\title{
DOA Tracking Algorithm for Acoustic Vector-Sensor Array via Adaptive PARAFAC-RLST
}

\author{
Weiyang Chen ${ }^{1,}$ a, Wei $\mathrm{Wu}^{1, \mathrm{~b}}$, Xiaoyu $\mathrm{Li}^{1, \mathrm{c}}$, and Xiaofei Zhang ${ }^{2, \mathrm{~d}^{*}}$ \\ ${ }^{1}$ College of Electronic and Information Engineering, Nanjing University of Aeronautics \& \\ Astronautics, Nanjing, China \\ 2 Jiangsu Key Laboratory of Internet of Things and Control Technologies (NanjingUniversity of \\ Aeronautics and Astronautics) \\ aweiweigenes@126.com, b39834290@qq.com, xiaoyuLi503@163.com, \\ dzhangxiaofei@nuaa.edu.cn
}

\begin{abstract}
Keywords: DOA tracking; acoustic vector-sensor array; PARAFAC-RLST
Abstract. In this paper, we consider a direction of arrival (DOA) tracking algorithm via adaptive parallel factor via recursive least squares tracking (PARAFAC-RLST) for acoustic vector-sensor array. The adaptive algorithm can efficiently track multiple targets by recursively updated the loading matrices at time $\mathrm{t}+1$ from their values at time $\mathrm{t}$, so that its global complexity is very lower than batch parallel factor (Batch-PAFARAC) without largely debasing angle estimation performance, as it does not require to compute the decomposition of 3rd-order tensor at time $\mathrm{t}+1$ by a batch algorithm. PARAFAC-RLST algorithm is more suitable for multiple targets DOA tracking, especially when direction angle cross each other. Simulation results verify the usefulness of our algorithm for DOA tracking.
\end{abstract}

\section{Introduction}

Acoustic vector sensor array is widely used in the sonar system and underwater acoustic communication system [1-3]. The direction of arrival (DOA) estimation is the core technology for acoustic vector sensor array [4-6]. A lot of DOA estimation algorithms for the acoustic vector sensor array are constantly emerging, including Capon algorithm [7], Multiple Signal Classification (MUSIC) algorithm [8, 9] ,Estimation of Signal Parameters via Rotational Invariance Techniques(ESPRIT) algorithm [10], Parallel Factor (PARAFAC) algorithm[11], Propagator (PM) algorithm [12].If subspaces are varying with time, they have to be tracked. But it will bring a large amount of computation for eigen-value decomposition of the cross correlation matrix or singular value decomposition of received data at every sampling instant, so they are not suitable for real-time applications. Over the past decade, adaptive tracking algorithms have been widely studied. The batch parallel factor (Batch-PAFARAC) in Refs.[13,14] requires the computation of three pseudo-inverses, and convergence often requires many cycles, even if the algorithm is well-initialized.

In this paper, we exploit parallel factor via adaptive recursive least squares tracking (PARAFAC-RLST) algorithm to track the DOA tracking for acoustic vector-sensor array, it is based on the recursive minimization of a weighted least-squares criterion. The adaptive PARAFAC-RLST algorithm used for Acoustic vector sensor array has not been addressed in the literature. We mainly discuss this algorithm in multiple target angles tracking, especially when their direction angles cross each other. It can efficiently track multiple targets when multiple targets are moving in acoustic vector sensor array, whose complexity is lower than batch-PAFARAC and PARAFAC-SDT [15]. Numerical experiments illustrate the accuracy and efficacy of the proposed algorithm in a variety of scenarios.

Notation: $(.)^{\mathrm{T}},(.)^{\mathrm{H}},(.)^{-1}$ and $(.)^{+}$denote transpose, conjugate-transpose, inverse, pseudo-inverse operations, respectively. $\mathrm{I}_{\mathrm{K}}$ is a $\mathrm{K} \times \mathrm{K}$ identity matrix; $\otimes$ and oare the Kronecker product, and Khatri-Rao product, respectively. 


\section{Data Model}

We consider an arbitrary acoustic vector sensor array system, and $M$ elements are arbitrarily distributed in the three-dimensional space, as shown in figure 1.

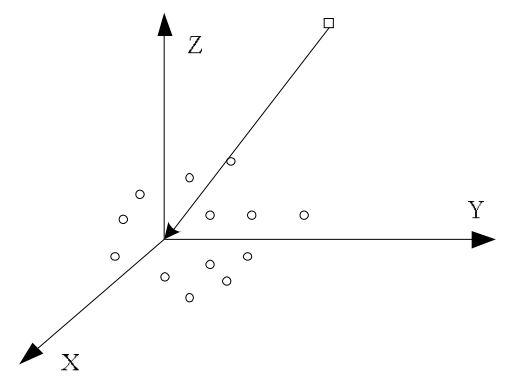

Fig.1 Arbitrary array model of the acoustic vector sensor [11]

We assume that there are a number of $\mathrm{K}(K<M)$ acoustic signals on the isotropic homogeneous fluid which incidence to the acoustic vector-sensor array from a two-dimensional DOA $\left(\boldsymbol{\theta}_{k}(t)=\left[\phi_{k}(t), \varphi_{k}(t)\right]\right)$, and at time $t$, collecting $\mathrm{J}$ snapshots, the output of the acoustic vector-sensor can be expressed as

$$
\mathbf{R}(t)=\boldsymbol{\Psi}(t) \mathbf{S}^{T}(t)+\mathbf{N}(t)
$$

where $\boldsymbol{\Psi}(t)=\mathbf{A}(t) \circ \mathbf{O H}(t), \mathbf{A}(t)=\left[\mathbf{a}_{1}(t), \mathbf{a}_{2}(t), \mathrm{L}, \mathbf{a}_{K}(t)\right], \mathbf{a}_{k}(t)=\left[q_{1}\left(\boldsymbol{\theta}_{k}(t)\right), q_{2}\left(\boldsymbol{\theta}_{k}(t)\right), \mathrm{L}, q_{M}\left(\boldsymbol{\theta}_{k}(t)\right)\right]^{T}, \mathbf{S}(t) \in \square^{J \times K}$, $\mathbf{N}(t) \in \square^{4 M \times J}$ is Gaussian white noise vector of zeros mean and covariance matrix $\sigma^{2} \mathbf{I}_{M N} \cdot \mathbf{R}(t)=[\mathbf{r}(1), \mathrm{L}, \mathbf{r}(J)], \mathbf{r}(t)=\Psi(t) \mathbf{s}(t)+\mathbf{n}(t), \mathbf{s}(t) \in \square^{K \times 1}$ is the vector of the complex envelops of the target signals.

\section{The Adaptive DOA Tracking Algorithm for Acoustic Vector-Sensor Array}

\section{A. PARAFAC Decomposition for Acoustic vector-sensor Array}

The PARAFAC decomposition of a tensor [15,16], $\chi \in \square^{M \times J \times N}$ is defined as

$$
\chi=\sum_{k=1}^{K} \mathbf{a}_{k} \mathbf{O b}_{k} \mathbf{o} \mathbf{c}_{k}
$$

where $\mathbf{a}_{k}, \mathbf{b}_{k}, \mathbf{c}_{k}$ are the kth columns of the loading matrices $\mathbf{A}_{T} \in \square^{M \times K}, \mathbf{S} \in \square^{\top \times K}, \mathbf{A}_{R} \in{ }^{M \rtimes K}$, respectively. We link the problem of acoustic vector-sensor array angle estimation with trilinear decomposition. According to its symmetry, we get the matrices as follows,

$$
\mathbf{R}^{(1)}=(\mathbf{A} \circ \mathbf{H}) \mathbf{S}^{T} ; \mathbf{R}^{(2)}=(\mathbf{S} \circ \mathbf{A}) \mathbf{H}^{T} ; \mathbf{R}^{(3)}=(\mathbf{H} \circ \mathbf{S}) \mathbf{A}^{T}
$$

\section{B. Basic Idea of Adaptive Tracking Algorithm}

Let us consider an estimate of the PARAFAC decomposition of a third-order tensor $\chi(t) \in \square^{M \times J(t) \times N}$, at time $t$

$$
\mathbf{R}^{(1)}(t) \square \boldsymbol{\Psi}(t) \mathbf{S}^{T}(t)
$$

At time $\mathrm{t}+1$, we can get [15]

$$
\mathbf{R}^{(1)}(t+1)=\left[\mathbf{R}^{(1)}(t), \mathbf{r}(t+1)\right]
$$

where $\mathbf{r}(t+1)$ is the new slice appended to $\mathbf{R}^{(1)}(t)$.

Let us consider a smooth variation of $\boldsymbol{\Psi}(t)$ between $t$ and $t+1$, i.e., $\Psi(t+1) \square \Psi(t)$, so we also have $\mathbf{S}^{T}(t+1)=\left[\mathbf{S}^{T}(t), \mathbf{s}^{T}(t+1)\right]^{T}$, where $\mathbf{s}^{T}(t+1)=\boldsymbol{\Psi}(t)^{+} \mathbf{r}(t+1)$. The least-squares update of $\boldsymbol{\Psi}(t+1)$ is then given by $\boldsymbol{\Psi}(t+1)=\mathbf{R}^{(1)}(t+1)\left(\mathbf{S}^{T}(t+1)\right)^{+}$.

Given $\boldsymbol{\Psi}(t+1)$, we can then re-update $\mathbf{r}(t+1)$ via substituting $\boldsymbol{\Psi}(t)$ by $\boldsymbol{\Psi}(t+1)$. Finally, we can get $\mathbf{A}(t+1)$ and $\mathbf{H}(t+1)$ from $\boldsymbol{\Psi}(t+1)$. 


\section{Truncated Window}

For a truncated window of length $\mathrm{W}(\mathrm{W}>\mathrm{K})$, we define weighted matrix

$$
\mathbf{R}_{T W}^{(1)}(t+1)=[\mathbf{r}(t+2-W), \mathrm{L}, \mathbf{r}(t), \mathbf{r}(t+1)]
$$

Define $\chi(t+1) \in \square^{M \times W \times N}$ expresses the tensor which is made of $\mathrm{W}$ latest slices, and we also have the weighted observed matrix $\mathbf{R}_{T W}(t+1)$, built from $\mathbf{R}_{T W}^{(1)}(t+1)$, and we can get the PARAFAC decomposition estimate of $\chi(t+1)$ via minimizing the least-squares criterion of the following truncated window as

$$
\min _{\left\{\mathbf{A}(t+1), \mathbf{S}_{T W}(t+1)\right\}}\left(\phi^{T W}(t+1)\right)
$$

where $\phi^{T W}(t+1)=\sum^{N} \lambda^{W-\tau} \square \mathbf{r}(\mu+\tau)-\mathbf{A}(t+1) \mathbf{O H}(t+1) \mathbf{s}^{T}(\mu+\tau) \square^{2}, \lambda$ is the forgetting factor, $\mu=t+1-W$ and $\mathbf{S}_{T W}(t+1)$ is made $\tau=$ of the last line $\mathrm{W}$ of $\mathbf{S}(t+1)$.

Then we can get weighted observation matrix $\mathbf{R}_{T W}(t+1)$ by $\mathbf{R}_{T W}^{(1)}(t+1)$

$$
\mathbf{R}_{T W}(t+1)=\mathbf{R}_{T W}^{(1)}(t+1) \Lambda
$$

where $\Lambda=\operatorname{diag}\left(\left[\lambda^{W-1 / 2}, \lambda^{W-2 / 2}, \mathrm{~L}, \lambda^{1 / 2}, 1\right]\right)$.

The update rule for $\mathbf{R}_{T W}^{(1)}(t)$ is

$$
\left[\lambda^{N / 2} \mathbf{r}(t+1-W), \mathbf{R}_{T W}(t+1)\right]=\left[\lambda^{1 / 2} \mathbf{R}_{T W}(t), \mathbf{r}(t+1)\right]
$$

\section{PARAFAC-RLST Algorithm}

PARAFAC-RLST is an adaptive tracking algorithm and follows the skeleton given in section B, which based on the minimization of a weighted least squares criterion [15].

Given an initial estimate of $\mathbf{s}(t+1)$, we can derive a recursive update for $\mathbf{\Psi}(t+1)$. Let $\nabla \phi^{T W}(t+1) \in \square^{4 M \times K}$ denote the gradient of $\phi^{T W}(t+1)$ with respect to $\Psi(t+1)$. So we have

$$
\begin{aligned}
\nabla \phi^{T W}(t+1)= & 2 \sum_{\tau=1}^{W} \lambda^{W-\tau}(\mathbf{r}(\mu+\tau)- \\
& \left.\mathbf{\Psi}(t+1) \mathbf{s}^{T}(\mu+\tau)\right) \mathbf{s}^{*}(\mu+\tau)
\end{aligned}
$$

After solving $\nabla \phi^{T W}(t+1)=0$, we can get

$$
\Psi(t+1)=\mathbf{D}_{T W}(t+1) \mathbf{P}_{T W}^{-1}(t+1)
$$

where $\mathbf{D}_{T W}(t+1)=\sum_{\tau=1}^{W} \lambda^{W-\tau} \mathbf{r}(\mu+\tau) \mathbf{s}^{*}(\mu+\tau), \mathbf{P}_{T W}(t+1)=\sum_{\tau=1}^{W} \lambda^{W-\tau} \mathbf{s}^{T}(\mu+\tau) \mathbf{s}^{*}(\mu+\tau)$.

And we can get $\mathbf{D}_{T W}$ and $\mathbf{P}_{T W}$ by recursive updates, so we have

$$
\begin{aligned}
& \mathbf{D}_{T W}(t+1)=\lambda \mathbf{D}_{T W}(t)+\mathbf{r}(t+1) \mathbf{s}^{*}(t+1)-\lambda^{W} \mathbf{r}(\mu) \mathbf{s}^{*}(\mu) \\
& \mathbf{P}_{T W}(t+1)=\lambda \mathbf{P}_{T W}(t)+\mathbf{s}^{T}(t+1) \mathbf{s}^{*}(t+1)-\lambda^{W} \mathbf{s}^{T}(\mu) \mathbf{s}^{*}(\mu)
\end{aligned}
$$

We may assume $\mathbf{Q}_{T W}=\mathbf{P}_{T W}^{-1}$ and apply matrix inversion lemma twice by utilising rank-2 update structure of $\mathbf{D}_{T W}$ and $\mathbf{P}_{T W}$, and then,

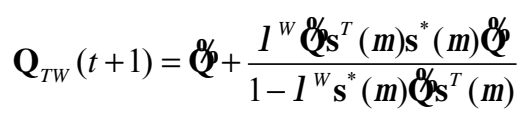

where $\& 0=\lambda^{-1} \mathbf{Q}_{T W}(t)-\frac{\lambda^{-2} \mathbf{Q}_{T W}(t) \mathbf{s}^{T}(t+1) \mathbf{s}^{*}(t+1) \mathbf{Q}_{T W}(t)}{1+\lambda^{-1} \mathbf{s}^{*}(t+1) \mathbf{Q}_{T W}(t) \mathbf{s}^{T}(t+1)}$

Finally, the update of $\boldsymbol{\Psi}(t+1)$ follows from the recursively updated matrices $\mathbf{D}_{T W}(t+1)$ and $\mathbf{P}_{T W}^{-1}(t+1)$.

$$
\Psi(t+1)=\mathbf{D}_{T W}(t+1) \mathbf{P}_{T W}^{-1}(t+1)
$$


Suppose now that, given the previous estimation of $\Psi(t+1)$, we want to estimate the last row $\mathbf{s}(t+1)$ of $\mathbf{S}(t+1)$. Since $\mathbf{A}^{+}(t+1)=\mathbf{P}(t+1) \mathbf{D}^{+}(t+1)$, the least-squares estimate of $\mathbf{s}(t+1)$ is given by

$$
\mathbf{s}^{T}(t+1)=\mathbf{P}_{T W}(t+1) \mathbf{D}_{T W}^{+}(t+1) \mathbf{r}(t+1)
$$

Our aim is now mainly to find a recursive update values of $\mathbf{D}_{T W}^{+}$and $\mathbf{P}_{T W}^{-1}(t+1)$ by utility their update structure,

$$
\begin{aligned}
\mathbf{D}_{T W}^{+}(t+1) & =\left(\lambda \mathbf{D}_{T W}(t)+\mathbf{r}(t+1) \mathbf{s}^{*}(t+1)-\lambda^{W} \mathbf{r}(t) \mathbf{s}^{*}(t)\right)^{+} \\
\mathbf{P}_{T W}^{-1}(t+1) & =\left(\lambda \mathbf{P}_{T W}(t)+\mathbf{s}^{T}(t+1) \mathbf{s}^{*}(t+1)-\lambda^{W} \mathbf{s}^{T}(u) \mathbf{s}^{*}(u)\right)^{-1}
\end{aligned}
$$

At this point, we can obtain estimates $\hat{\mathbf{A}}(t+1)$ and $\hat{\mathbf{H}}(t+1)$ of $\mathbf{A}(t+1)$ and $\mathbf{H}(t+1)$ from the above steps. Then we can obtain the angle of each source by using the rotation invariance of the matrix at time $t+1$.

According to the received noise signal, $\hat{\mathbf{H}}=\mathbf{H} \Pi \mathbf{\Delta}+\mathbf{N}$ can be obtained through the decomposition, where $\boldsymbol{\Pi}$ is the switching matrix, $\boldsymbol{\Delta}$ is the dimension fuzzy matrix, $\mathbf{N}$ is the estimated error matrix. We can get the matrix $\hat{\mathbf{H}}$ by normalizing the matrix $\hat{\mathbf{H}}, \hat{\mathbf{h}}_{\mathrm{i}}$ is the $i$ th column of $\hat{\mathbf{H}}$. In the absence of noise, $\hat{\mathbf{h}}_{\mathbf{i}}$ can be expressed as

$$
\hat{\mathbf{h}}_{\mathbf{i}}=\left[\begin{array}{c}
1 \\
\cos \phi_{i} \cos \varphi_{i} \\
\sin \phi_{i} \cos \varphi_{i} \\
\sin \varphi_{i}
\end{array}\right]
$$

We can get a two-dimensional angle as

$$
\begin{gathered}
\hat{\varphi}_{i}=\arcsin \left[\hat{\mathbf{h}}_{\mathbf{i}}(4)\right] \\
\hat{\phi_{i}}=\arctan \left[\hat{\mathbf{h}}_{\mathbf{i}}(3) / \hat{\mathbf{h}}_{\mathbf{i}}(2)\right]
\end{gathered}
$$

But the calculated angle did not associate the source, it must be use related technology to accurately track the source. In this paper, we use angle association ideology in Refs [9] or other related technical perspective to link the DOA for acoustic vector sensor array.

Suppose that, the angle of each source is known or has been estimated by other algorithms at time $t$. Elevation and azimuth respectively are $\varphi_{1}(t), \varphi_{2}(t), \mathrm{L}, \varphi_{K}(t)$ and $\phi_{1}(t), \phi_{2}(t), \mathrm{L}, \phi_{K}(t)$. Construct the signal covariance matrix $\hat{\Gamma}, \hat{\Gamma}=\hat{\mathbf{\Psi}}^{+}(\boldsymbol{\theta}(t+1)) \hat{\mathbf{\Psi}}(\boldsymbol{\theta}(t)) \mathbf{S} \hat{\boldsymbol{\Psi}}^{H}(\boldsymbol{\theta}(t+1))\left[\hat{\boldsymbol{\Psi}}^{+}(\boldsymbol{\theta}(t+1))\right]^{H}$, find out the location $\left(j_{1}, k_{1}\right)$ of the absolute maximum element in this matrix, and get rid of the row and column of this absolute value, then find out the location $\left(j_{2}, k_{2}\right)$ of the absolute maximum element in the remaining elements. And so on, until find out the position of the $K$ th element. According to the location of each maximal element, we can associate the angle estimate of its corresponding source.

Till now, the major steps of DOA tracking of adaptive PARAFAC-RLST algorithm for acoustic vector-sensor array are given as follows:

Step1: Create an initial estimation $\mathbf{s}^{T}(t+1)$ for $\mathbf{S}^{T}$.

Step2: According to (12), (13), (17) and (18), update $\mathbf{D}, \mathbf{P}, \mathbf{D}^{+}, \mathbf{P}^{-1}$ by applying matrix inversion lemma twice.

Step3: Re-estimate $\mathbf{s}^{T}(t+1)$ by (16), and obtain $\mathbf{S}^{T}(t+1)$.

Step4: Update $\mathbf{\Psi}(t+1)$, and then get estimate values $\mathbf{A}(t+1)$ and $\mathbf{H}(t+1)$ from $\mathbf{\Psi}(t+1)$.

Step5: Obtain the corresponding DOA at that moment according to the estimate of $\mathbf{A}(t+1)$ and $\mathbf{H}(t+1)$.

Step6: Associate the estimated DOA.

Step7: Repeat Step 1-6, track the angle estimate for the next moment. 


\section{E. Complexity analysis}

In contrast to batch-PARAFAC algorithm in the Ref. [14], PARAFAC-RLST has a low computational load as it avoids computing the decomposition of 3rd-order tensor at time $\mathrm{t}+1$ by a batch algorithm. The major computational complexity of PARAFAC-RLST is $K^{2}(16 M N+72)+K(144 M N+10 N+20)$, while batch-PARAFAC algorithm needs $88 K^{3}+64 K^{2}(W N+M N+M W)+8 K(3 M N W+W N+M N+M W)$.

\section{Simulation Results}

We exploit adaptive PARAFAC-RLST algorithm in this paper to consider targets emitting uncorrelated narrow band signals to verify its effectiveness. We define RMSE

$$
R M S E=\frac{1}{K} \sum_{k=1}^{K} \sqrt{\frac{1}{Q} \sum_{q=1}^{Q} \frac{1}{T} \sum_{t=1}^{T}\left[\left(\hat{\phi}_{k, q, t}-\phi_{k, q, t}\right)^{2}+\left(\hat{\varphi}_{k, q, t}-\varphi_{k, q, t}\right)^{2}\right]}
$$

where $\hat{\phi}_{k, q, t}$ is the estimate of the azimuth $\phi_{k}$ of the $q$ th Monte Carlo trial at time $t, \hat{\varphi}_{k, q, t}$ is the estimate of the elevation $\varphi_{k}$ of the $q$ th Monte Carlo trial at time $t$.

Notation: $M, J, K$ and $Q$ are the number of array elements, the snapshots, sources and Monte Carlo trials, respectively. $N$ is a fixed value, $\mathrm{N}=4$. The length of a truncated window is $W . M=8, N=6, J=100$, $K=3, W=10, Q=1000$. The forgetting factor $\lambda$ is 0.97 . Duration of the tracking procedure, $\mathrm{T}$ new slices will be observed and $\mathrm{T}=200$. The sampling interval is set at $1 \mathrm{~s}$.

Simulation 1: Figs.2-4 presents the tracking results of adaptive PARAFAC-RLST algorithm under different DOA change trajectory. We set signals to noise rate (SNR) for $15 \mathrm{~dB}$. We can know obviously the adaptive algorithm is able to work to track DOA effectively for acoustic vector-sensor array.

Simulation 2: This simulation analyzes algorithm performance according to the DOA trajectory of Figs.2-3, and the following simulation parameters are the same. Fig.5 shows DOA tracking performance comparison between our algorithm and batch PARAFAC algorithm. It is indicated that this algorithm has close angle estimation performance to batch PARAFAC algorithm, while the complexity of this algorithm is far lower than the batch - PARAFAC algorithm.

Simulation 3: Fig.6 shows DOA tracking performance of the adaptive algorithm with different $M$, $J=100, K=3$. It is clear that the angle estimation performance of adaptive algorithm is improved as the number of antennas increases.

Simulation 4:Fig.7 investigates the tracking performance of adaptive PARAFAC-RLST algorithm with different values of $\mathrm{K}, \mathrm{J}=100, \mathrm{M}=8$. From Fig. 7 we find that angle estimation performance of our algorithm degrades with the increasing of $\mathrm{K}$. It is suggests that the interference between sources will reduce when the number of sources decreases.



Fig.2 The DOA tracking performance of adaptive algorithm, $\mathrm{SNR}=15 \mathrm{~dB}$



Fig.3 The DOA tracking performance of adaptive algorithm, $\mathrm{SNR}=15 \mathrm{~dB}$ 


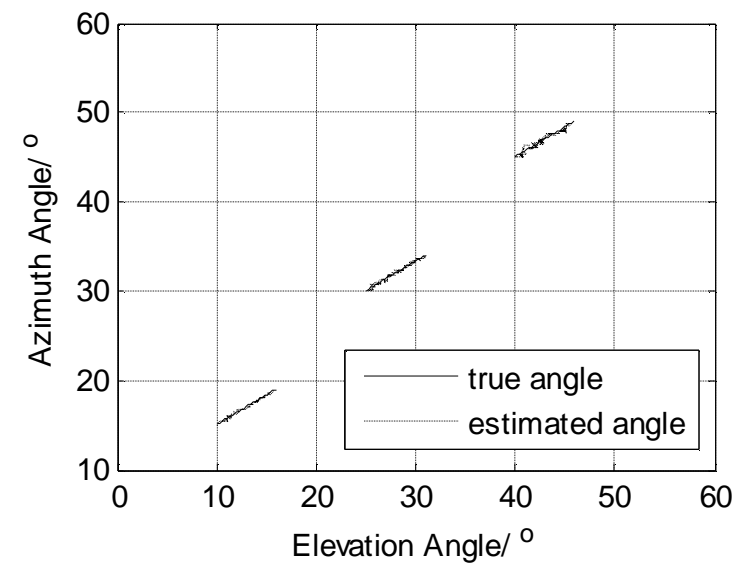

Fig.4 The DOA tracking performance of adaptive algorithm, $\mathrm{SNR}=15 \mathrm{~dB}$



Fig.6 The DOA tracking performance with different values of $M$

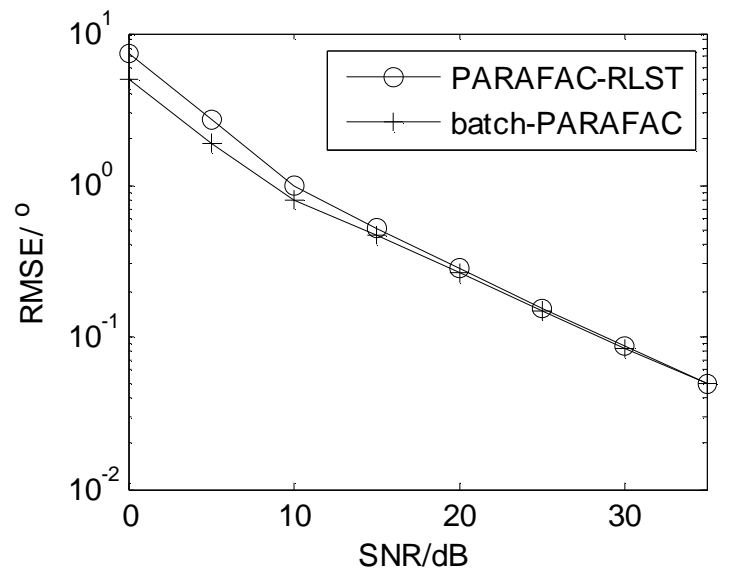

Fig. 5.The DOA tracking performance comparison of different algorithms

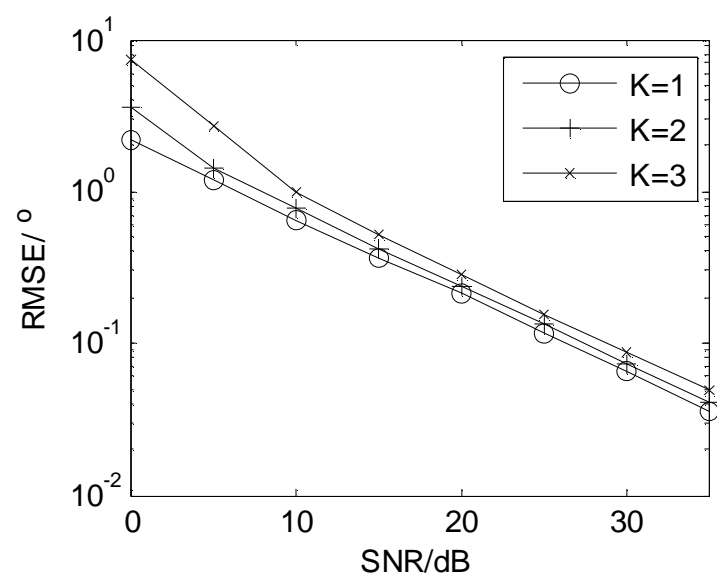

Fig.7 The DOA tracking performance with different values of $K$

\section{Conclusions}

In this paper, the problem of angle tracking for acoustic vector sensor array using adaptive PARAFAC-RLST algorithm has been addressed. The adaptive PARAFAC-RLST algorithm has close angle tracking performance to batch-PARAFAC, while the complexity load of adaptive PARAFAC-RLST algorithm is much less than that of it. The adaptive PARAFAC-RLST algorithm is more suitable for DOA tracking for acoustic vector sensor array, and numerical experiments illustrate the accuracy and efficacy of adaptive algorithm.

\section{Acknowledgements}

This work is supported by China NSF Grants $(61371169,61301108,61471191,61471192$, 61271327), Qing Lan Project, and priority academic program development of Jiangsu high education institutions.

\section{References}

[1] Nehorai A, Paldi E. Acoustic vector-sensor array processing[J]. IEEE Transactions on Signal Processing, 1994, 42(9):2481 - 2491.

[2] Wu Y I, Wong K T, Lau S. The Acoustic Vector-Sensor's Near-Field Array-Manifold[J].IEEE Transactions on Signal Processing, 2010, 58(7):3946 - 3951. 
[3] Liu Z, Ruan X, He J. Efficient 2-D DOA estimation for coherent sources with a sparse acoustic vector-sensor array[J]. Multidimensional Systems and Signal Processing, 2013, 24(1):105-120.

[4] HAW KESM, N EHORA IA. A coustic vector-sensor beam forming and Capon direction estimation [J].IEEE Tran s on Signal Process, 1998, 46 (9):2291-2304.

[5] HOCHWALD B, N EHORA IA. Identifiability in the processing models with vector-sensor applications [J]. IEEE Tran s on Signal Process, 1996, 44 (1):83-95.

[6] Palanisamy P, Kalyanasundaram N, Swetha P M. Two-dimensional DOA estimation of coherent signals using acoustic vector sensor array[J]. Signal Processing, 2012, 32 (1):19-28.

[7] YU Ming. Acoustic Vector-Sensor Capon Direction Estimation [D]. Harbin Engineering University, 2004. DOI:10.7666/d.y670097.

[8] LIANG Guolong,ZHANG Kai, Performance evaluation of DOA estimation using a single acoustic vector-sensor based on an improved MUSIC algorithm[J], Journal of Harbin Engineering University,2012,33(1):30-36

[9] YAO Zhixiang, HU Jinhua, YAO Dongming, A bearing estimation algorithm using an acoustic vector sensor array based on MUSIC[J], Acta Acustica,2008,33(4):305-309.

[10]J. He, S. Jiang, J. Wang, Z. Liu. Direction finding in spatially correlated noise fields with arbitrarily-spaced and far-separated subarrays at unknown locations [J]. IET Radar, Sonar \& Navigat. , 2009, 3(3):278-284.

[11]CHEN Weiyang, DOA Estimation for Acoustic Vector-sensor Array[D], Nanjing University of Aeronautics and Astronautics,2011.

[12] GU Chen,HE Jin,ZHU Xiao-hua, Extended-Aperture Two-Dimensional DOA Estimation with Acoustic Vector Sensor Array Using the Propagator Method[J], Acta Electronica Sinica,2010,10: 2377-2382.

[13]R.Bro, PARAFAC: Tutorial and applications, Chemom. Intell. Lab. Syst., vol.38, pp. 149-171, 1997.

[14]N. D. sidiropoulos, G. B. Giannakis, and R. Bro, Blind PARAFAC receivers for DS-CDMA systems, IEEE Trans. Signal Process., vol. 48,pp. 810-823,2000.

[15]D. Nion and N. D. Sidiropoulos, Adaptive Algorithms to Track the PARAFAC Decomposition of a Third-Order Tensor, IEEE. Trans. on Signal Processing, vol. 57, no. 6, June 2009

[16] S.L.Campbell and C.D.Meyer, Generalized Inverses of Linear Transformations [M], New York: Dover, 1991. 